\title{
The STARE/SABRE story
}

\author{
E. Nielsen ${ }^{1}$ and W. Schmidt ${ }^{2}$ \\ ${ }^{1}$ Max Planck Institute for Solar System Research, Justus-von-Liebig-Weg 3, 37077 Göttingen, Germany \\ ${ }^{2}$ Finnish Meteorological Institute, Earth Observation, P.O. Box 503, 00101 Helsinki, Finland
}

Correspondence to: E. Nielsen (nielsen@mps.mpg.de)

Received: 23 May 2013 - Revised: 29 January 2014 - Accepted: 4 February 2014 - Published: 7 March 2014

\begin{abstract}
In January 1977 a new type of radar aurora experiment named STARE (Scandinavian Twin Aurora Radar Experiment) commenced operation in northern Scandinavia. The purpose of the experiment was twofold: to make observations of the nature of radar auroras, and to contribute to the study of solar-terrestrial relationships (or space weather). The experiment was designed for automatic continuous operation, and for nearly two and a half decades it provided estimates of electron flows with good spatial coverage and resolution and good time resolution. It was a successful experiment that yielded a wealth of observations and results, pertaining to, and based on, the observed time variations of the electron flows and to the spatial flow pattern observed at any given time. This radar system inspired the creation of a similar system, SABRE (Sweden And Britain Radar Experiment), which increased the field of view towards the southwest of STARE. This system commenced operation in 1982.
\end{abstract}

\section{Introduction}

In most of the plasma regions in the space around the Earth, the electrons and ions are, to a very good approximation, moving under the influence of electric and magnetic fields in an $E \times B$ drift. This is, for example, the case in the $F$-region ionosphere, and in general at altitudes above $130 \mathrm{~km}$. On the other hand, at altitudes below $90 \mathrm{~km}$, the mobility of both ions and electrons is controlled by collisions with the neutral gas. However at altitudes in the $E$-region, from 90 to $130 \mathrm{~km}$, only the mobility of ions is dominated by the neutrals, while electrons are $E \times B$ drifting. This completely changes the characteristics of the plasma from that at higher and lower altitudes. At $E$-region altitudes, instabilities are excited in order to create plasma waves, which can be observed with coherent radar techniques from the ground (see, for example, Nielsen (1988a), where radar principles and some properties of radar signals backscattered from the ionosphere are introduced, followed by presentation of data acquisition, coherent detection, digital sampling and data processing).

The auroral radar radio signal is backscattered from the ionosphere $E$-region by electrostatic ion waves excited by the two-stream (Buhneman, 1963; Farley, 1963) or gradientdrift (Rogister and D'Angelo, 1970) plasma instabilities. The waves are excited preferably in directions perpendicular to the geomagnetic field lines. Towards the middle of the 1970s there was emerging experimental evidence and perception that the backscattered signal, propagating horizontally and nearly perpendicular to the geomagnetic field lines, had a double-pulse Doppler shift related to the electron drift velocity component along the near-horizontal radar line of sight to the backscatter volume. In principle, this raises the possibility of estimating the electron drift velocity in a common volume by combining Doppler velocities measured in this common volume from at least two different directions. Ecklund et al. (1975) were able to study the drift velocity of radar aurora irregularities at the point of intersection of two narrow-beam radars. Greenwald and Ecklund (1975) observed Doppler velocities as large as $2000 \mathrm{~m} \mathrm{~s}^{-1}$, and argued that these are two-stream irregularities with a velocity determined by the electron drift speed. Ecklund et al. (1977) compared $F$-region plasma drift velocities with radar aurora drift velocities and observed remarkably good agreement.

The new radar aurora radar system as proposed by Ray Greenwald (Greenwald et al., 1978) is an expansion on this basic idea of stereoscopic Doppler shift measurements. The proposed system consists of two radar stations separated by a large distance (hundreds of kilometers) typically at a 


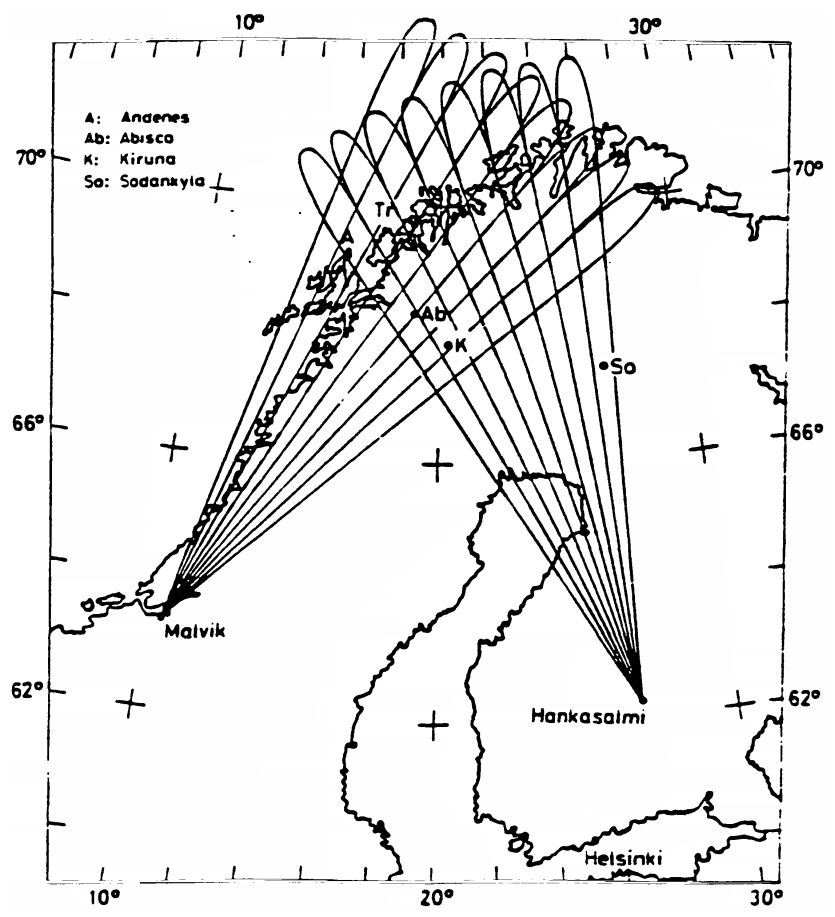

Figure 1. The STARE radar stations in Norway and Finland, with eight narrow antenna lobes from the two stations. The STARE field of view covers a geomagnetic latitude range from 65 to $70^{\circ}$.

geomagnetic latitude of around $66^{\circ}$ (Fig. 1). The nearly horizontal, generally poleward-directed narrow antenna beams of the two radars cross over a large common "area" in the ionospheric $E$-region in the auroral zone. In this configuration the radar wave vector is nearly perpendicular to the geomagnetic field lines, as is required for a good radar auroral backscatter signal. With several narrow beams together with a range resolution along each narrow beam of, for example, $15 \mathrm{~km}$, there now exists not one but several hundred points of intersections in which Doppler velocity data from the two radars can be measured and combined to yield the irregularity drift velocity. This allows, for the first time, derivation of the irregularity drift velocity over a large area with high spatial and temporal resolution. In this way, drift velocities can typically be derived over a field of view of $400 \mathrm{~km} \times 400 \mathrm{~km}$ with a spatial resolution of approximately $20 \mathrm{~km} \times 20 \mathrm{~km}$ and $20 \mathrm{~s}$ time resolution.

\section{STARE}

In 1974 the new director at MPAe (Max Planck Institute for Aeronomy), Germany, Prof. Ian Axford invited Ray Greenwald to come to MPAe, starting 1975, to design, build and operate his proposed system with a field of view in the auroral zone over northern Scandinavia, centered near Tromsö. MPAe has since been renamed to MPS (Max Planck Institute for Solar System Research). The system was to be part of

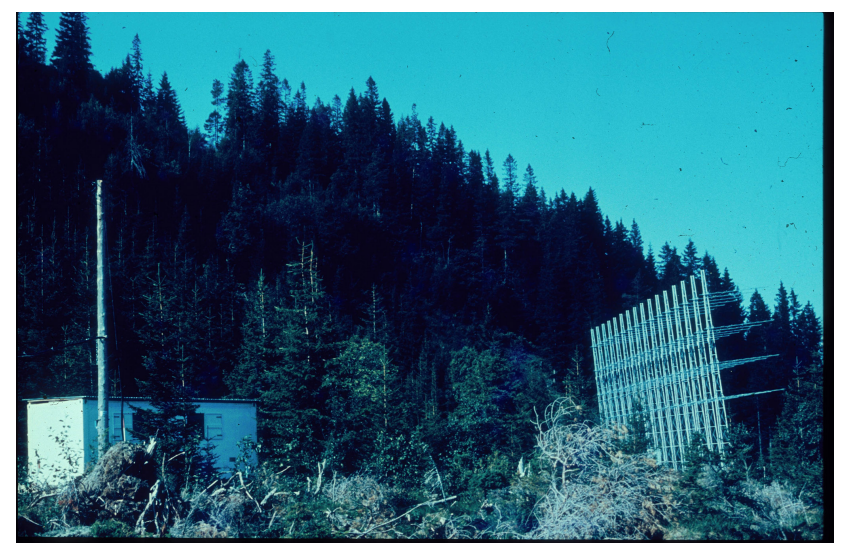

Figure 2. Receiving array (about $30 \mathrm{~m}$ length) and container with radar.

the network of ground-based ionospheric experiments being installed at this time for operation during the IMS (International Magnetospheric Study), including

- SMA (Scandinavian Magnetometer Array), covering northern Scandinavia;

- EISCAT (European Incoherent SCATter facility);

- the Ionosphere Heating Facility, located near Tromsö;

- the narrow-beam riometer, also near Tromsö;

- optical measurements.

The STARE field of view was to further include the rocket launch sites at Andoya and Kiruna, as well as the magnetic footprint of the geosynchronous satellite GEOS 2. The field of view also occasionally coincided with observations by orbiting satellites such as TRIAD and DE1.

The technical design of a radar station was devised by Ray Greenwald in close cooperation with Werner Weiss, an experienced radio wave engineer at MPAe. Erling Nielsen joined the experiment as a postdoctoral associate in 1976. The multiple narrow beams were formed by a receiving array consisting of 64 Yagi antennas, distributed on 16 masts separated by a wavelength, and on each mast were 4 stacked Yagis also separated by a wavelength (Fig. 2). The outputs from each mast were fed into a 16-element Butler matrix, a passive phasing network that simultaneously forms 16 narrow lobes (Fig. 3).

A detailed description of the STARE radar system can be found in Greenwald et al. (1978). The two radar stations in the STARE system were located in Malvik (originally at $10.73^{\circ} \mathrm{E}$ and $63.67^{\circ} \mathrm{N}$, after 1995 at $10.754^{\circ} \mathrm{E}$ and $\left.63.426^{\circ} \mathrm{N}\right)$, Norway, and Hankasalmi $\left(26.6494^{\circ} \mathrm{E}\right.$ and $62.3047^{\circ} \mathrm{N}$ ), Finland (Fig. 1). The local contact in Norway was Prof. Tor Hagfors, University of Trondheim. SINTEF at the University of Trondheim was responsible for the local management of the radar operations in Malvik $(140.0 \mathrm{MHz})$, 


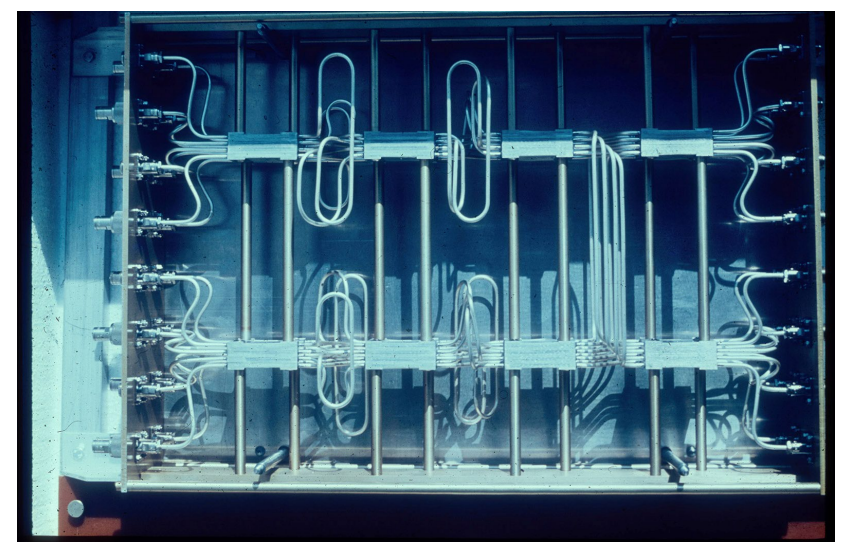

Figure 3. The Butler matrix is a passive phasing network used with the receiving array to form the multi-narrow-lobe pattern.

while the Hankasalmi radar (143.8 MHz) was managed by Risto Pellinen at the Finnish Meteorological Institute (FMI) in Helsinki. The local work on the radars was carried out by Tore Barlindhaug, Norway, and Teppo Rinkinen, Finland, during the entire duration of the experiment.

The radar stations were designed for automatic operations under computer control using a Data General NOVA 2 computer. The software was written in assembly code without the benefit of a higher-level language. The radar stations were operated in a single-pulse-double-pulse mode. The single pulse yielded the backscatter intensity over the field of view, and the double pulse yielded the radial Doppler velocity over the field of view in measurement points (beam/range) where the intensity was better than $2 \mathrm{~dB}$ over noise. The observation data were written to $800 \mathrm{BPI}$ magnetic tape at the end of each integration time. The data tapes from the two stations were sent to MPAe for merging of the Doppler velocity measurements to yield the drift velocities over the field of view. Later, Mario Ierkic (Arecibo Observatory) developed software to measure the autocorrelation of the backscattered signal and calculate the spectrum. This was done by measuring the backscattered signal for a series of sequential double pulses with pulse separation increasing from 200 to $2000 \mu$ s. These observations could only be carried out with the station manned. The first analysis program was also in assembly code, but by the fall of 1980 a multiuser Data General system with Fortran capability and with a large data storage device was installed at MPAe, which considerably eased the data analysis work.

Shortly after the system was put into operation, it became evident that the irregularity drift velocity was closely related to the ionospheric electron drift velocity, or electric field. The field of view of the STARE system covers the rocket trajectory of the typical rocket launch from Andenes. The system was put into operation on 17 January 1977, and on 23 January a rocket was launched with an electric field detector in the payload (Cahill et al., 1978). At this time the radar sta-

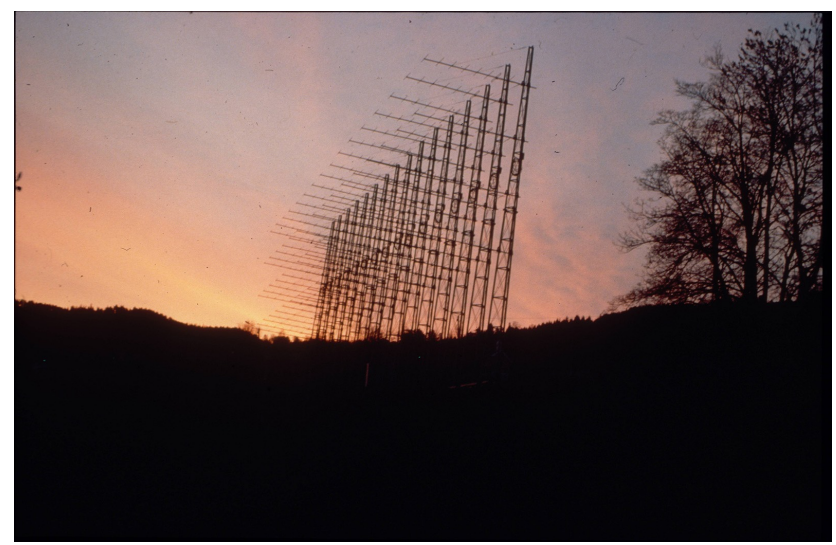

Figure 4. STARE receiver array at the new site in Norway.

tion in Malvik was manned and had a telephone connection to the rocket launch control room in Andoya. The data display at the station showed that a region separating an area of positive Doppler velocities from an area of negative Doppler (convection reversal) was drifting slowly equatorward approaching the location of apogee of the rocket trajectory. It was therefore possible to predict the time of launch to ensure the rocket would fly across the convection reversal and make measurements of the reversing electric field. The good agreement of the drift velocity and electric field measurements was the first indication that the system operated as expected. This was also the first good example of simultaneous rocket-radar observations.

Possibly the most directly convincing experimental evidence and irrevocable sign of a close relationship between the ionospheric electric field and the irregularity drift velocity came from an unexpected source. A surprising (or nonpredicted) result of the STARE radar measurements was the observation of toroidal and poloidal wave fluctuations of the ionospheric electron drifts. These fluctuations had amplitude and phase variations across the field of view as were predicted to be associated with geomagnetic micropulsations. The observations with STARE followed the predictions of theory in detail, implying that the drift velocities were closely connected to the ionospheric electric field fluctuations. This important result was first seen and described by Walker et al. (1979).

Thus, it was confirmed early on that the irregularity drifts derived from STARE Doppler measurements were related to the electron drift velocity in the $E$-region. It remained to be determined just how good an approximation to the electron drift the irregularity drift was. This has been a subject of intense study throughout the duration of the STARE project. It proved to be a difficult problem to resolve, because the irregularity drift depends on many parameters of the plasma, parameters that are hard to reliably estimate. Initially it was believed that the irregularity drift component measured by a radar in the linear approximation was given by the cosine 
to the angle (flow angle) between the drift velocity and the radar $\boldsymbol{k}$ vector (Greenwald et al., 1978). This turned out to be a good approximation only for large flow angles, where the backscatter is from secondary wave activity. At smaller flow angles the irregularity drift was limited in magnitude, but, importantly, owing to secondary plasma activity, the limiting drift was a function of the electron drift speed. This made it possible to correct for the limitation of the Doppler velocity and regain the energy dependence of the electron drift velocity (Nielsen and Schlegel, 1983, 1985). More studies on the subject of the relationship between irregularity and electron drift can be found in Sect. 5 .

\section{SABRE}

The success of the STARE system convinced the British science community to invest in a radar system that would expand the field of view of the STARE system. The new radar system was a STARE-type system with two radar stations built on the STARE model. One station $(153.2 \mathrm{MHz})$ was in Wick, Scotland, built and operated by the University of Leicester under the leadership of Prof. Tudor Jones, and one station (142.585 MHz) in Uppsala, Sweden, was built and operated by MPAe in cooperation with Uppsala Ionospheric Observatory; here, Prof. Rolf Boström and Aage Hedberg were responsible for the local management of the radar operations. The British team made a major modification to the transmitter, rebuilding it around small ceramic tubes and replacing the expensive klystron used in the original design. The British radar had additional features that allowed for real-time control of the radar and transfer of data in real time from the Wick station to the University of Leicester.

The station locations were chosen such that the new fields of view were to the southwest of the STARE system, expanding the latitude and longitude coverage of the STARE radars. The new system (Nielsen et al., 1983a) was denoted SABRE (Sweden And Britain Radar Experiment); it commenced operation in 1982 and ended in 1989. At that time the Uppsala station was relocated to MPAe, where it was used in the development of the ambitious "New STARE" update of the STARE system.

An important advantage of the SABRE field of view was that it allowed, in a small region, for simultaneous observations in a common backscatter volume with three antenna lobes, from Uppsala, Hankasalmi and Malvik. This allowed for a more detailed analysis of the radial irregularity velocities (Nielsen et al., 1983b).

\section{New STARE}

By the end of the 1980s there was demand for improving the temporal and spatial resolution of the STARE system, especially in the region over EISCAT, and heating for the purpose of joint plasma physics studies. At the same time, spare parts for the transmitter electronics became unavailable, leaving only the option of either shutting down the radar permanently or replacing it with a state-of-the-art design.

In 1987 it was agreed between the then MPAe in Katlenburg-Lindau, Germany, and FMI in Helsinki, Finland, to transfer ownership of the Hankasalmi station to FMI and to jointly continue the cooperation of STARE as well as jointly undertake an upgrade of the system. At a planning meeting on 13 January 1988 it was decided that the new STARE concept should include a freely programmable radar controller, allowing for the implementation of complex multipulse schemes for high spatial resolution, and a separate hardware correlator, providing the computational speed needed to process the complex radar echoes that would exceed the capabilities of standard PC-type computers of that time. MPAe was to be responsible for developing the radar controller and for refurbishing the radar electronics, receivers and transmitters, while FMI was responsible for developing the hardware correlator and for developing the computer and data network environment.

From the beginning of May 1988, Dr. Walter Schmidt was employed by FMI to lead the New STARE project as project manager at FMI. Prior to this he had worked at the European Incoherent Scatter radar facility (EISCAT) in Kiruna, Sweden, responsible for the development of its control system software and later the day-to-day operation of the tristatic ionospheric radar.

Between July and September 1988 the Norwegian station in Malvik was switched off to allow for the transmitter and receiver to be upgraded, while Hankasalmi continued operation as monostatic radar until 20 February 1989, when it also was switched off and the receiver shipped to MPAe for refurbishment and integration with the new transmitter, radar controller and PC. This system was reinstalled in Hankasalmi beginning of May. By the end of 1989 the new system, linked to the national Finnish data network and including the new correlator, was basically operational and was used during the following years for several monostatic observations, while the second correlator, shipped to MPAe in spring 1990 and intended for the Malvik station, never worked despite several extensive repair efforts.

At a meeting at MPAe in the summer of 1991 with several participants from MPAe and FMI and chaired by Prof. Dr. Tor Hagfors, director at MPAe, it was decided that work on the failing hardware correlator was to be stopped and the system replaced with a simpler concept developed at MPAe as a recovery solution without the hardware correlator. The ambitious alternating code phase modulation plans were given up as the computational power of the simplified environment was not sufficient for its implementation. The new system foresaw an additional PC with a DOS operating system for control of the radar operations. The hardware correlator was replaced with a bank of now commercially available fast ADC cards for the DOS PC. The data preprocessing (correlations) was done by software in real time. Only the radar 
controller remained unchanged, allowing the implementation of simple multipulse schemes for autocorrelation/spectrum measurements alongside the single- and double-pulse usage. The Unix-based computer was reconfigured to act primarily as recipient of the radar data files from the DOS PC, and otherwise to act as a networking interface and a remote monitoring and data archiving system. These modifications were implemented in both STARE stations. The new remote monitoring possibilities of the system allowed for routine data quality checks from the home institutes, automatic data transfer from the radar sites to the institutes without the need for shipping physical data storage media, and the implementation of a real-time monitoring link from the ESRANGE launch site for ionospheric sounding rockets.

Dr. Johan Silén, who was also a former EISCAT staff member, was named as the new project manager at FMI in 1991.

After a relocation of the Norwegian station (Fig. 4 shows the new receiver array), made necessary by an expansion of Malvik's housing area, the bistatic radar resumed its operation in November 1995 after an interruption of more than $6 \mathrm{yr}$. While initially mainly special campaigns were run, continuous routine data are available online starting from 9 October 1997 onwards. STARE continued until 17 May 2005, when it was finally shut down after more than two decades of successful observations.

\section{Some contributions}

The purpose of this section is to outline the great variety of applications of STARE/SABRE observations and to list some of the results of the measurements. What particularly distinguishes these new radar systems is their ability to estimate electron flows in the high-latitude ionosphere $E$-region over a large area with good spatial resolution, as well as their ability to measure these flow patterns as a function of time with good time resolution.

The electric fields in the ionosphere are fundamentally imposed by the interaction of the solar wind with the Earth's atmosphere and magnetic field. The interaction gives rise to one of the most fundamental processes in space plasma physics: magnetospheric convection. The ionosphere is part of the magnetosphere, and thus observations of plasma flows in the ionosphere open a window through which we can study the interactions of the solar wind with our planet. The wide latitudinal coverage and the continuous operation (over $24 \mathrm{~h}$ ) allows for observations of the global high-latitude electron flow patterns. Drift velocities as a function of latitude were averaged over $10 \mathrm{~min}$ intervals and their variations examined as a function of latitude and magnetic local time. Both radar systems, STARE and SABRE, separately and together, have been used to study the dependence of the ionosphere plasma flows on geomagnetic activity. It was found that for increasing magnetic activity, the morning convection cell increased with time, the reversal from westward to eastward electron drifts and vice versa occurred earlier, the largest electric field magnitudes were observed during the afternoon, and the average location at which the largest fields are observed moved to lower latitudes. The flows during quiet times and modifications for increasing activity were in reasonable agreement with theoretical predictions (Zi and Nielsen, 1982; Waldock et al., 1985).

Mishra and Nielsen (1996) qualitatively studied the occurrence of electric fields larger than $20 \mathrm{mV} \mathrm{m}^{-1}$ in the ionosphere in the STARE field of view for variations of the interplanetary magnetic field north-south $\left(B_{z}\right)$ and east-west $\left(B_{y}\right)$ components. For $B_{z}<0$ they found the occurrence of these strong electric fields increased as the magnitude of $\left|B_{z}\right|$ increased. For an increasingly large southward-directed magnetic field, the occurrence of backscatter (large electric fields) was more likely to be observed, and it covered a larger percentage area of the STARE field of view. When $B_{z}$ was positive, i.e., northward directed, the occurrence was likely to be much smaller and cover a smaller percentage area. For $B_{z}>0$ the occurrence of backscatter was dependent on the sign of $B_{y}$, clearly displaying a dawn-dusk asymmetry of plasma flows for these conditions.

Magnetic field-aligned currents provide an important energy link between the solar wind and the magnetosphere/ionosphere. Measuring of field-aligned currents with coherent radars was first attempted by Sofko et al. (1995). Data sets similar to those used in convection flow studies above were used by Kosch and Nielsen (2001) to derive the field-aligned currents from the STARE/SABRE observations of ionospheric electric fields (electron flows). Considering that small-scale conductivity gradients and electric field variations due to auroral precipitation are effectively averaged away, it was shown that the currents are determined by the product of Pedersen conductivity and the divergence of the observed electric fields. In practice, computation of the divergence of the electric field involves integrating the perpendicular component of the field vector around a closed loop. In this case the loop is the small square centered on the grid points in latitude and local time (longitude) of the observation data. Using a realistic Pedersen conductivity model, the high-latitude global distribution of field-aligned currents could be calculated with high spatial resolution. Results were obtained for low and for high magnetic activity, as well as for negative and positive orientation of the $z$ component of the interplanetary magnetic field. This is the first extensive study of field-aligned currents using coherent radar observations. The results compare very favorably to other ground-based and satellite measurements.

One of the major sinks of solar wind energy is ionospheric Joule heating caused by dissipation of energy by the Pedersen currents in the ionospheric (closure of field-aligned currents). STARE/SABRE averaged flow velocity data sets in a latitude versus magnetic local time framework were also used by Kosch and Nielsen (1995) to estimate averaged 
high-latitude Joule heating. Calculation of the Joule heating involves the product of Pedersen conductivity and the square of the electric field magnitude. Realistic Pedersen conductivity models were used together with the observed electric fields for varying magnetic activity and interplanetary magnetic fields. It was found for the first time that the ionospheric electric field magnitude as well as Joule heating increase with increasingly negative $z$ component of the interplanetary magnetic field. This is the first such study using coherent radar observations. The latitude and diurnal variation of Joule heating as well as the estimated mean hemispherical heating of $170 \mathrm{GW}$ are in good agreement with earlier results.

Wilkinson et al. (1986) demonstrated how one can derive estimates of the ionospheric Hall conductance by combining ground magnetometer measurements with STARE/SABRE spatial electron flow observations. The ground magnetic field is the result of divergence-free (closed) currents in the ionosphere. In the absence of conductance gradients, the ionospheric currents closing field-aligned currents are equal to the Pedersen currents. This is equivalent to considering the Hall current divergence-free and that is therefore responsible for the total ground magnetic response arising from ionospheric currents. The Hall current is determined by the product of an assumed Hall conductance and the electron flow velocities, observed with STARE. Assuming a constant Hall conductance and summing over the Hall current map, using the Biot-Savart law, the expected ground magnetometer response can be calculated (Walker et al., 1979). The actual Hall conductance is determined by the best fit of the magnetometer data and the ground magnetic prediction based on STARE measurements. The derived Hall conductance shows seasonal and diurnal behavior similar to empirical conductance models. Conductance gradients cause the source-free and closure currents to have Hall and Pedersen contributions, respectively. Examples in which the calculated magnetic response was rotated relative to the magnetic observations could be interpreted as evidence of spatial conductance gradients.

Ionospheric currents are often estimated using ground magnetometers and magnetometer arrays. This approach has one difficulty - namely, that, at least in principle, all the ionospheric currents will contribute to the detected magnetic field, not only the overhead current. The magnetometer measurement, and thus the equivalent current, is a point measurement; however it is an average of all contributions from ionospheric currents at the location of the magnetometer. This means in general that the observed ground magnetometer data can be matched by several different assumed ionosphere current systems. This creates important restraints on the analysis of magnetometer observations by themselves. Baumjohann et al. (1980) showed that simultaneous twodimensional observations of ground magnetic fields (SMA) and ionospheric electric fields (STARE) have basic advantages over previous observations when studying the threedimensional current flow. Inhester et al. (1981) used such observations (SMA, STARE and all-sky camera measurement) to study the westward traveling surge at the substorm expansion phase. The basic approach was to determine the spatial variations of several parameters: (1) electric charge from the optical measurements, (2) ionosphere electric fields from STARE, (3) conductance from the literature, (4) the total ionosphere current from electric fields and conductance, (5) field-aligned currents from the divergence of the total current, and (6) the predicted ground equivalent current is calculated using a Biot-Savart integration over the horizontal and field-aligned currents. The parameters were then adjusted within realistic limits to yield the best fit between calculated and observed ground currents (equivalent currents). It was conclusively shown that there is an upward field-aligned current in the head of the surge of $50000 \mathrm{~A}$ intensity. The STARE and SMA measurements supplemented each other perfectly, and the combination of the two projects turned out to be very productive and lead to many master's and doctoral theses.

Another study, involving analysis of individual STARE frames with superposed all-sky camera data and riometer observations, concerned the electron flows during the equatorward surge and followed by the poleward-moving, intense auroral arc during the substorm expansion phase (Nielsen, 1991). The arc coincides with the narrow region of energetic electron precipitation observed with the narrow-beam riometer in Tromsö (Nielsen and Axford, 1977). Nielsen and Greenwald (1978) found that the poleward expansion of the activated energetic arc could not be explained by ionospheric $E \times B$ drift. No case could be found in which the electric field had a strong eastward component. They suggested instead that the poleward motion is a forced motion: it is the result of anti-sunward propagation of the region in which oppositely directed tail magnetic fields are merged during substorm expansion. Electrons are accelerated in the merging region and propagate along the newly reconnected magnetic field lines to increasing latitudes in the ionosphere. This is an example where STARE/SABRE measurements yield insight into processes deep in the magnetosphere. Nielsen et al. (1999) and Goertz et al. (1985) showed other examples that illustrate how electron flow signatures in the ionosphere reflect processes far away on the magnetopause: flux transfer events. These events involve a line current flowing between the ionosphere and the flux transfer region and are associated with an observed swirl in the ionospheric flows.

The STARE/SABRE experiments turned out to be a very important tool for observations of geomagnetic micro pulsation activity. This was first noted and demonstrated by Walker et al. (1979). Observations of the north-south and east-west electric field component over a large area with good spatial and temporal resolution make these radar systems especially well suited for studying pulsation phenomena. In each point of measurements a time series of the electric field components is available, and a Fourier analysis, or equivalent, can be carried out to determine spatial 
variations of the pulsation amplitude and phase at the pulsation frequency. This is important input for a theoretical understanding of the pulsation. Different types of pulsations have been observed. Some events (Walker, 1980) are monochromatic with resonance pulsation, maximum amplitude and zero phase at the magnetic shell with eigenfrequency equal to the detected frequency (Nielsen and Allan, 1983). TRIAD observations of field-aligned currents confirmed the field-aligned currents predicted from STARE observations (Poulter et al., 1982). Villain (1982) showed the polarization ellipse changed across the resonance region as expected for a resonance toroidal-mode hydromagnetic wave in the magnetosphere. The observations are consistent with generation of the pulsations by the Kelvin-Helmholz instability on the magnetopause, excited by passage of the solar wind. Other events (Poulter and Nielsen, 1982) are wide band with magnetic shells oscillating at their eigenfrequency in a wide latitude range. While monochromatic events can last for hours, these events are transient, having an impulsive onset and lasting typically only three to five cycles. These pulsations are excited when a discontinuity in the solar wind hits the magnetosphere and sets it ringing at its eigenfrequencies. Since the energy input to the pulsations is impulsive and short lasting, the pulsations are relatively quickly attenuated owing to Joule heating losses in the ionosphere (Nielsen, 2001). Other events (Allan et al., 1982; Walker et al., 1982) have been observed for which neither the period nor amplitude nor phase changes with latitude. These events are often coincident with particle flux oscillations at geosynchronous orbit. The observations have been interpreted in terms of the drift mirror instability. Further observed were eastward-drifting Pc5 pulsations (Walker and Nielsen, 1984), Ps6 pulsations with eastward-drifting omega bands (Mravlag et al., 1991; Nielsen and Sofko, 1982), Pi2 pulsations (Sutcliffe and Nielsen, 1992) and PG (giant) pulsations (Poulter et al., 1983).

To examine whether it is possible to excite $1 \mathrm{~m}$ electron density irregularities in the auroral ionosphere, HF radio waves from the powerful Ionospheric Heater Facility near the center of the STARE field of view were directed overhead in the ionospheric $E$-region. Creation of irregularities was detected by the appearance of backscatter in the STARE radar measurements (Hibberd et al., 1983). Analysis of a large amount of measurements (Hoeg et al., 1986) revealed that irregularities are more likely to occur for $O$-mode heating than for $X$-mode heating. The $e$-folding growth time was a few seconds. The phase velocities appear to be controlled by the background electric field. The frequency spectrum of the signal backscattered from the artificial irregularities was obtained using the multipulse operation of the STARE system. The results were presented and discussed by Nielsen and Rietveld (2003). We have also used the radar systems to observe the properties of the backscattered signal from natural ionosphere irregularities. The intensity of the signal backscattered was found to be a strongly decreasing func- tion of the aspect angle, the angle between the radar $\boldsymbol{k}$ vector and the direction of the geomagnetic field. It was possible to determine the attenuation with aspect angle separately for primary and secondary waves (Nielsen, 1988b). Aspect angle dependence of the mean Doppler velocity was also observed. To account for the observations, an electron-neutral collision frequency a factor of 5 larger than the nominal value was required (Nielsen, 1986). This result is consistent with other analyses of STARE/SABRE Doppler velocity data (Reinleitner and Nielsen, 1985) and is in line with theoretical developments. Observations of power spectra of the backscattered signal from natural radar aurora were presented and discussed by Haldoupis et al. (1984), Nielsen et al. (1984) and Haldoupis and Nielsen (1989).

Throughout the operations of the STARE/SABRE systems there was an interest in testing, and if possible improving, the accuracy with which electron drift velocities are derived from radar measurements. The drift velocities derived from STARE/SABRE Doppler velocities (electric fields) have been favorably compared with field measurements using other techniques (Nielsen and Whitehead, 1983): on rockets (electric field), balloons (electric field), three-station Doppler measurements using STARE/SABRE (Nielsen et al., 1983b), at the SMA (equivalent currents) and at the Ionospheric Heater Facility. (VLF radio waves can be generated in the lower ionosphere by periodically modulating the natural currents using high-power HF radio waves. Such waves have been generated and detected on the ground such that the amplitude, proportional to the electric field in the current region, could be reconstructed (Barr et al., 1985).) There was general good agreement for velocities $<700 \mathrm{~m} \mathrm{~s}^{-1}$ (or electric fields $<35 \mathrm{mV}$ ). For larger fields the estimates tended to be limited in magnitude. Eventually, Doppler velocities observed with STARE were compared to electron drift velocities observed with the incoherent scatter facility, EISCAT, located near the center of the STARE field of view (Nielsen and Schlegel, 1985). The measurements in the $E$ - and $F$-region could, with great accuracy, be related to the same magnetic flux tube. It was found that the Doppler velocities of electron density irregularities produced by the two-stream instability in the $E$-region are limited to a value near the ion acoustic velocity. Importantly, it was moreover found that this value is a strong increasing function of the electron drift velocity (electric field), in agreement with theoretical predictions (St.-Maurice et al., 1981). An algorithm was developed that enabled the use of STARE/SABRE data for deriving estimates of the electron drift velocity, which are in excellent agreement with EISCAT velocity data. This procedure especially improved the velocity estimates for large electric fields - work on this is still ongoing. Uspensky et al. (2003) examined the importance of off-orthogonality and ion motion on the STARE velocities, and Uspensky et al. (2004) studied westward electron flows. The analysis showed that the double-pulse velocities are consistently smaller than the multipulse velocities in the evening sector at large flow angles. 
This is especially puzzling, because for observations in the morning sector, the double-pulse and multipulse velocities do not differ significantly. Nielsen (2004) discussed the observed mean velocities measured with the double-pulse technique for increasing time lag between the pulses. Uspensky et al. (2005) proceeded to utilize multipulse power spectra observations with a view to further improve the drift velocity estimates one can derive from STARE data.

Furthermore, there is a potential possibility that the observed irregularity velocities varies with backscatter altitude. To test this, the Norwegian radar was operated as a vertical interferometer in order to measure vertical variations of electron density fluctuations in the $E$-region. This was accomplished by placing an outrigger array in front of the STARE receiver array in Malvik (11 $\mathrm{m}$ distance). The radar was operated in a mode that allowed for measurements of the crosscorrelation coefficients between the outrigger and several lobes of the receiver array. This provided, for the first time, the capability to study the backscatter altitude and dynamic height variations. It was concluded that useful information on backscatter altitude can be obtained during homogenous conditions (for both eastward and westward flow). The results show the mean backscatter layer altitude and width can undergo regular variations that are often closely related to concurrent changes observed in the echo intensity and irregularity velocity (Ierkic et al., 1992).

\section{Summary}

Until beginning of the 1970s, radar auroral research was directed mainly towards understanding the physics of ionosphere plasma in the 90 to $130 \mathrm{~km}$ altitude range at high (polar) and equatorial latitudes. The purpose was to experimentally describe the plasma and theoretically account for the observations. There was a limited group of scientists engaged in such studies; it was a specialist field of research. However, the discovery that the backscattered radar signals carried information on the ionospheric electric field, and, furthermore, that this information could be extracted by a remote sensing instrument as a radar, meant that radar auroral work became of direct interest to scientists in many other fields of geophysical studies. The reason for this is that the electric field, imposed on the Earth's plasma environment by the interaction of the solar wind with the planet, is the most fundamental parameter controlling the plasma environment. The electric field is important for a wide range of geophysical studies of the ionosphere to the far reaches of the magnetosphere. A STARE-type system uses remote sensing radar technique to measure estimates of the electric field as a function of time in selected points in the ionosphere. Importantly, a further aspect is that the remote detection capability also for a given time measures the field pattern over a large field of view $\left(160000 \mathrm{~km}^{2}\right)$. Thus, the measurement technique allows distinguishing between spatial and true temporal variations. A single-point ground-based experiment would not allow this, and a rocket or satellite experiment measures the field along the trajectory with no possibility of determining whether observed variations are spatial and/or temporal in nature. The fundamental nature of the parameter measured by the radar experiment and the ability to distinguish between temporal and spatial variations have made the STARE/SABRE system a very successful ground-based experiment. This has been internationally recognized and led to widespread interest in the measurements.

Acknowledgements. We thank W. I. Axford and T. Hagfors, directors at MPAe whose underlying support from start to finish was essential and invaluable. Thanks are also due to the technical staff at MPAe, FMI and the University of Leicester (W. Güttler, P. Janhunen and C. Thomas) and at the radar stations at Malvik, Uppsala and Hankasalmi (T. Barlindhaug, A. A. Hedberg and T. Rinkinen) for their successful efforts to keep the radars in operation. We are deeply grateful to R. Pellinen, director at FMI; Prof. T. Jones of the University of Leicester; and Prof. R. Boström of Uppsala Observatory for directing and managing the STARE/SABRE operations in Finland, the United Kingdom and Sweden. We also want to thank the innumerable scientists who made observations in northern Scandinavia for making their data available for joint studies with STARE/SABRE. Special thanks are due to all the fellow scientists, who came from all over the world - from the USA, Russia, China, India, New Zealand, Australia, South Africa, Canada, the United Kingdom, France, Norway, Sweden, Denmark, Finland and Greece - to Katlenburg-Lindau in Germany to work on STARE/SABRE observations. Their varied background, enthusiasm and hard work contributed greatly to the scientific exploitation of the measurements and to the success of the project.

Edited by: R. J. Pellinen

Reviewed by: T. R. Robinson, R. Greenwald, F. Honary, and two anonymous referees

\section{References}

Allan, W., Poulter, E. M., and Nielsen, E.: STARE observations of a Pc5 pulsation with large azimuthal wave number, J. Geophys. Res., 87, 6163-6172, 1982.

Barr, R., Rietveld, M. T., Kopka, H., Stubbe, P., and Nielsen, E.: ELF radiation from the polar electrojet antenna, Nature, 317, 155-157, 1985.

Baumjohnn, W., Untiedt, J., and Greenwald, R. A.: Joint twodimensional observations of ground magnetic and ionospheric electric fields associated with auroral zone currents. 1. Three dimensional current flows associated with a substorm-intensified eastward electrojet, J. Geophys. Res., 85, 1963-1978, 1980.

Buhneman, O.: Excitation of field-aligned sound waves by electron streams, Phys. Rev. Lett., 10, 285-287, 1963.

Cahill, L. J., Greenwald, R. A., and Nielsen, E.: Auroral radar and rocket double probe observations of the electric field across the Harang discontinuity, Geophys. Res. Lett., 5, 687-690, 1978.

Ecklund, W. L., Balsly, B. B., and Greenwald, R. A.: Crossed beam measurements of the diffuse aurora, J. Geophys. Res., 80, 18051114, 1975. 
Ecklund, W. L., Balsly, B. B., and Carter, D. A.: A preliminary comparison of F-region plasma drifts and E-region irregularity drifts in the auroral zone, J. Geophys, Res., 82, 195-197, 1977.

Farley, D. T.: A Plasma instability resulting in field-aligned irregularities in the ionosphere, J. Geophys. Res., 68, 6083-6097, 1963.

Goertz, C. K., Nielsen, E., Korth, A., Glassmeier, K. H., Haldoupis, C., Hoeg, P., and Hayward, D.: Observations of a possible ground signature of flux-transfer events, J. Geophys. Res., 90, 40694078, 1985.

Greenwald, R. A. and Ecklund, W. L.: A new look at radar auroral motions, J. Geophys. Res., 80, 3642-3648, 1975.

Greenwald, R. A., Weiss, W., Nielsen, E., and Thomson, N. R.: STARE: A new radar auroral backscatter experiment in northern Scandinavian, Radio Sci., 13, 1021-1039, 1978.

Haldoupis, C. and Nielsen, E.: Very large phase velocities of non two-stream, meter scale irregularities in the high latitude $\mathrm{E}$ region ionosphere, J. Geophys. Res., 94, 13489-13504, 1989.

Haldoupis, C., Nielsen, E., and Ierkic, H. M.: STARE Doppler spectral studies of westward electrojet radar aurora, Planet. Space Sci., 32, 1291-1300, 1984

Hibberd, F. H., Nielsen, E., Stubbe, P., and Kopka, H.: Production of auroral zone E-region irregularities by ionospheric heating, J. Geophys. Res., 88, 6347-6351, 1983.

Hoeg, P., Nielsen, E., Stubbe, P., and Kopka, H.: Heater Induced 1Meter Irregularities, J. Geophys. Res., 91, 11309-11320, 1986.

Ierkic, H. M., Haldoupis, C., Moorcroft, D. R., and Nielsen, E.: Coherent radar interferometry of vertical irregularity structures in the auroral E region, Radio Sci., 27, 743-758, 1992.

Inhester, B., Baumjohann, W., Greenwald, R. A., and Nielsen, E.: Joint two-dimensional observations of ground magnetic and ionospheric electric fields associated with auroral zone currents - 32. Auroral zone currents during the passage of a westward travelling surge, J. Geophys. Res., 49, 155-162, 1981.

Kosch, M. J. and Nielsen, E.: Coherent radar estimates of average high-latitude ionospheric Joule heating, J. Geophys. Res., 100, 12201-12215, 1995.

Kosch, M. J. and Nielsen, E.: Statistical average estimates of high latitude field-aligned currents from the STARE and SABRE coherent VHF radar system, Adv. Space Res., 27, 1239-1244, 2001.

Marvlag, E., Scourfield, M. W. J., Walker, A. D. M., Sutcliefe, P. R., and Nielsen, E.: Simultaneous observations of omega band related phenomena in both hemispheres, J. Atmos. Terr. Phys., 53, 309-317, 1991

Mishra, S. P. and Nielsen, E.: Response of ionospheric electric fields to variations in the interplanetary magnetic field, Ann. Geophys., 14, 794-802, doi:10.1007/s00585-996-0794-z, 1996.

Nielsen, E.: Aspect angle dependence of mean Doppler velocities of 1-m auroral plasma waves, J. Geophys. Res., 91, 10173-10177, 1986.

Nielsen, E.: Coherent radar techniques, in: World Ionosphere/Thermosphere Study, WITS Handbook, Vol. 2, edited by: Liu, C. H., SCOSTEP Secret, Urbana/USA, 285-315, 1988a.

Nielsen, E.: Aspect angle dependence of backscatter intensity of 1-m auroral plasma waves, J. Geophys. Res., 93, 4119-4124, 1988 b.

Nielsen, E.: VII-3. Ionosphere-magnetosphere mapping of dynamic auroral structures during substorms, in: Auroral Physics, edited by: Meng, C.-I., Rycroft, M. R., and Frank, L. A., Syndicat of the University Press Cambridge, Cambridge, United Kingdom, 409-418, 1991.

Nielsen, E.: Direct experimental test of Joule heating damping of a Pc5 pulsation using the STARE radar system, J. Geophys. Res., 106, 25835-23845, 2001.

Nielsen, E.: Mean velocities measured with the double pulse technique, Ann. Geophys., 22, 3531-3536, doi:10.5194/angeo-223531-2004, 2004.

Nielsen, E. and Allan, E.: A double-resonance Pc5 pulsation, J. Geophys. Res., 88, 5760-5764, 1983.

Nielsen, E. and Axford, W. I.: Small scale auroral absorption events associated with substorms, Nature, 267, 502-504, 1977.

Nielsen, E. and Greenwald, R. A.: Variations in ionospheric currents and electric fields in association with absorption spikes during the substorm expansion phase, J. Geophys. Res., 83, 5645-5654, 1978.

Nielsen, E. and Rietveld, M. T.: Observations of backscatter autocorrelation functions from 1.07-m ionospheric irregularities generated by the European Incoherent Scatter Heater Facility, J. Geophys. Res., 108, A5, doi:10.1029/2002JA009537, 2003.

Nielsen, E. and Schlegel, K.: A first comparison of STARE and EISCAT electron drift velocity measurements, J. Geophys. Res., 88, 5745-5750, 1983.

Nielsen, E. and Schlegel, K.: Coherent radar Doppler measurements and their relationship to the ionospheric electron drift velocity, J. Geophys. Res., 90, 3498-3504, 1985.

Nielsen, E. and Sofko, G. J.: Ps6 spatial and temporal structure fom STARE and riometer observations, J. Geophys. Res., 87, 81578165, 1982.

Nielsen, E. and Whitehead, J. D.: Radar auroral observations and ionospheric electric fields, Adv. Space Res., 2, 131-144, 1983.

Nielsen, E., Güttler, W., Thomas, E. C., Stewart, C. P., Jones, T. B., and Hedberg, L. A.: A new radar auroral backscatter experiment, Nature, 304, 712-714, 1983a.

Nielsen, E., Whitehead, J. D., Hedberg, L. A., and Jones, T. B.: A test of the cosine relationship using three-radar velocity measurements, Radio Sci., 18, 230-236, 1983 b.

Nielsen, E., Haldoupis, C., Fejer, B. G., and Ierkic, H. M.: Dependence of auroral power spectra variations upon electron drift velocity in the eastward electrojet, J. Geophys. Res., 89, 253-260, 1984.

Nielsen, E., Bruns, M., Pardowitz, I., Perplies, H., Bemmann, L., Janhunen, P., and Huuskonen, A.: STARE: Observations of a field-aligned line current, Geophys. Res. Lett., 26, 21-24, 1999.

Poulter, E. M. and Nielsen, E.: The hydromagnetic oscillation of individual shells of the geomagnetic field, J. Geophys. Res., 87, 10432-10438, 1982

Poulter, E. M., Nielsen, E., and Potemra, T. A.: Field-aligned currents associated with Pc5 pulsations: STARE and TRIAD observations, J. Geophys. Res., 87, 2331-2336, 1982.

Poulter, E. M., Allan, W., Nielsen, E., and Glassmeier, K.-M.: STARE radar observations of a PG pulsation, J. Geophys. Res., 88, 5668-5676, 1983.

Reinleitner, L. A. and Nielsen, E.: Self-consistent analysis of electron drift velocity measurements with the STARE/SABRE system, J. Geophys. Rs., 90, 8477-8486, 1985.

Rogister, A. and D'Angelo, N.: Type 2 irregularities in the equatorial electrojet, J. Geophys. Res., 75, 3879-3887, 1970. 
Sofko, G. J., Greenwald, R. A., and Bristow, W.: Direct determination of large-scale magnetospheric field-aligned currents, Geophys. Res. Lett., 22, 15, 2041-2044, 1995.

St.-Maurice, J.-P., Schlegel, K., and Banks, P. M.: Anomalous heating of the polar E-region by unstable plasma waves, 2 . Theory, J. Geophys. Res., 86, 1453-1462, 1981.

Sutcliffe, P. R. and Nielsen, E.: The ionospheric signature of Pi 2 pulsations observed by STARE, J. Geophys. Res., 97, 1062110636, 1992.

Uspensky, M., Koustov, A., Janhunen, P., Pellinen, R., Danskin, D., and Nozawa, S.: STARE velocities: the importance of offorthogonality and ion motions, Ann. Geophys., 21, 729-743, doi:10.5194/angeo-21-729-2003, 2003.

Uspensky, M., Koustov, A., Janhunen, P., Nielsen, E., Kauristie, K., Amm, O., Pellinen, R., Opgenoorth, H., and Pirjola, R.: STARE velocities: 2. Evening westward electron flow, Ann. Geophys., 22, 1077-1091, doi:10.5194/angeo-22-1077-2004, 2004.

Uspensky, M., Koustov, A., Sofieva, V., Amm, O., Kauristie, K., Schmidt, W., Nielsen, E., Pulkinen, T., Pellinen, R., Milan, S., and Ajola, R.: Multipulse and double-pulse velocities of Scandinvian Twin Auroral Radar Experiment (STARE) echoes, Radio Sci., 40, RS3008, doi:10.1029/204RS003151, 2005.

Villain, J. P.: Characteristics of Pc5 micropulsations as determined with the STARE experiment, J. Geophys. Res., 87, 129-139, 1982.
Waldock, J. A., Jones, T. B., and Nielsen, E.: Mean auroral E-region plasma convection patterns measured by SABRE, Nature, 313, 204-206, 1985.

Walker, A. D. M.: Modelling of Pc5 pulsation structure in the magnetosphere, Planet. Space Sci., 28, 213-223, 1980.

Walker, A. D. M. and Nielsen, E.: STARE observations of an eastward propagating Pc5 pulsations with large azimuthal wave number, Geophys. Res. Lett., 11, 259-262, 1984.

Walker, A. D. M., Greenwald, R. A., Stuart, W. F., and Green, C. A.: STARE auroral radar observations of Pc5 geomagnetic pulsations, J. Geophys. Res., 84, 3373-3388, 1979.

Walker, A. D. M., Greenwald, R. A., Korth, A., and Kremser, G.: STARE and Geos 2 observations of a storm time Pc5 ULF pulsation, J. Geophys. Res., 87, 9135-9146, doi:10.1029/JA087iA11p09135, 1982.

Wilkinson, P. J., Nielsen, E., and Lühr, H.: Ionospheric conductances: Associations between ionospheric E region electric field and ground magnetometer variations, J. Geophys. Res., 91, 5839-5849, 1986.

Zi, M. and Nielsen, E.: Spatial variations of electric field in the high latitude ionosphere, J. Geophys. Res., 87, 5202-5206, 1982. 